\title{
EIGENFUNCTIONS OF PLANE ELASTOSTATICS III THE WEDGE
}

\author{
V. T. BUCHWALD
}

(received 1 August 1964)

\begin{abstract}
Summary
The boundary value problem of the infinite wedge in plane elastostatics is reduced to the solution of a differential-difference equation. The complementary function of this equation is determined in the form of a Fourier integral, which, on expansion by residue theory, gives the complete eigenfunction expansion for the wedge. The properties of the eigenfunctions are discussed in some detail, and an orthogonality property is derived.

The problem of the wedge loaded on its faces is shown to correspond to the task of finding by Fourier transforms particular integrals of the differential-difference equation. The case of an isolated normal load on one face of the wedge is taken as a particular example, and the appropriate eigenfunction expansions are obtained.
\end{abstract}

\section{Introduction}

Problems of the wedge in plane elastostatics have previously been considered from two points of view. On one hand, Tranter [1], and Green and Zerna [2] have given the solution of the problem of the wedge, loaded on its faces, in terms of certain Fourier integrals. On the other hand, eigenfunction expansions of the Airy stress function have, in effect, been used by Carrier and Shaw [3], and Morley [4], to solve certain boundary value problems connected with regions with corners.

The chief aim of this paper is to give a unified account of boundary value problems of the wedge. Complex variable techniques developed in a previous paper [5] are used to reduce the problem to the general solution of a differential-difference equation. The complex potentials are expressed in terms of certain Fourier integrals, which, on evaluation by residue theory give the relevant eigenfunction expansions. The closure of the expansions can be established in this way, and the significance of the isolated load and couple at the vertex is explained in terms of the eigenvalue zero.

If $\alpha$ is the semi-vertical angle of the wedge, where $0<\alpha<\pi$, the case in which $\alpha$ is the first root of the equation 


$$
\tan 2 \alpha=2 \alpha,
$$

is exceptional, since two of the eigenvalues are equal. Appropriate forms of the eigenfunction expansion can be deduced in this case by evaluating the residues at the corresponding second order poles.

\section{The Differential-Difference Equation}

Using the notation of Green and Zerna [2] for plane strain, the stress components in polar coordinates are given by

$$
\Theta(z, \bar{z})=\tau_{r r}+\tau_{\theta \theta}=2\left[\Omega^{\prime}(z)+\bar{\Omega}^{\prime}(\bar{z})\right],
$$

and

$$
\Phi(z, \bar{z})=\tau_{\theta \theta}-i \tau_{r \theta}=\Omega^{\prime}(z)+\bar{\Omega}^{\prime}(\bar{z})+\bar{z}{\overline{\Omega^{\prime \prime}}}^{\prime}(\bar{z})+\frac{\bar{z}}{z} \bar{\omega}^{\prime}(\bar{z}),
$$

where $\Omega(z), \omega(z)$, are functions of the complex variable $z$ which are analytic in the regions considered. The bars indicate complex conjugate functions and variables in the usual way. The complex displacement is given by

$$
2 \mu D=\kappa \Omega(z)-z \bar{\Omega}^{\prime}(\bar{z})-\bar{\omega}(\bar{z}),
$$

where $\mu$ is Lame's constant, and $\kappa=3-4 \sigma$, where $\sigma$ is Poisson's ratio.

Assume that isotropic material occupies the interior of the wedge $-\alpha<\arg z<\alpha$, and that the faces arg $z= \pm \alpha$ of the wedge are loaded by given stresses such that only plane deformations occur. The problem is to find $\Omega(z), \omega(z)$, analytic in the interior of the wedge, given that

$$
\Phi= \begin{cases}f_{0}(r), & z=r e^{i \alpha} \\ g_{0}(r), & z=r e^{-i \alpha}\end{cases}
$$

where $f_{0}(r), g_{0}(r)$, are prescribed given functions of $r$.

Consider the conformal mapping
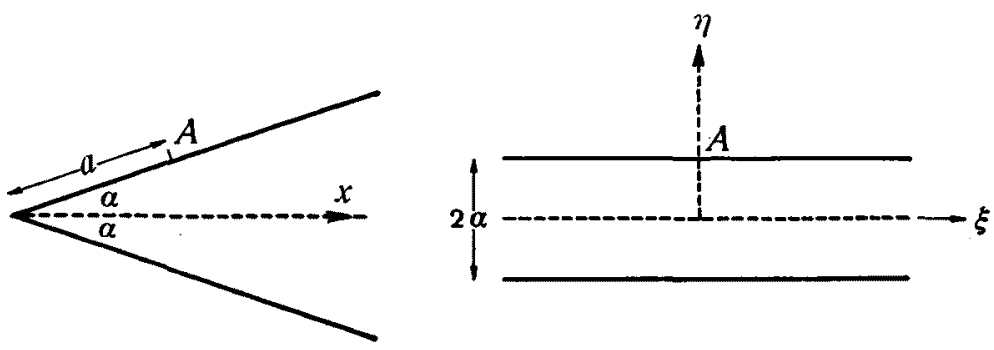

Fig. 1. 


$$
z=a e^{5}, \quad r=a e^{\xi}, \quad \theta=\eta,
$$

where $\zeta=\xi+i \eta, z=r e^{i \theta}$, and $z(d / d z)=d / d \zeta$. The interior of the wedge is mapped onto the strip $S,|\eta|<\alpha,-\infty \leqq \xi \leqq \infty$, as in Fig. 1. The boundary conditions $(\mathbf{2 . 4})$

are changed to

$$
\Phi= \begin{cases}f(\xi), & \eta=\alpha, \\ g(\xi), & \eta=-\alpha,\end{cases}
$$

where $f, g$, are easily obtained from $f_{0}, g_{0}$, and $\Phi$ is given in the new variables by

$$
a \Phi(\zeta, \bar{\zeta})=e^{-\zeta}\left[\Omega^{\prime}(\zeta)+\bar{\omega}^{\prime}(\bar{\zeta})\right]+e^{-\bar{\zeta}} \bar{\Omega}^{\prime \prime}(\bar{\zeta}) .
$$

We shall denote by $S^{U}$ the strip $\alpha<\eta<3 \alpha$, and by $S^{L}$ the strip $-3 \alpha<\eta<-\alpha$. Let the analytic continuation of $\Omega(\zeta)$ into $S^{U}$ be given by

$$
\Omega_{v}^{\prime}(\zeta)=-e^{2 i \alpha} \bar{\Omega}^{\prime \prime}(\zeta-2 i \alpha)-\bar{\omega}^{\prime}(\zeta-2 i \alpha),
$$

for $\zeta$ in $S^{U}$. Note that since $\Omega(\zeta)$ is analytic for $\zeta$ in $S$, it follows that $\Omega(\zeta-2 i \alpha)$ is analytic for $\zeta$ in $S^{U}$, so that $\Omega^{\prime}(\zeta)$, as defined in (2.8), is analytic for $\zeta$ in $S^{U}$. Replacing $\zeta-2 i \alpha$ by $\bar{\zeta}$ in (2.8), we find, for $\zeta$ in $S$,

$$
\bar{\omega}^{\prime}(\bar{\zeta})=-e^{2 i \alpha} \bar{\Omega}^{\prime \prime}(\bar{\zeta})-\Omega_{U}^{\prime}(\bar{\zeta}+2 i \alpha) .
$$

Substitution of this expression for $\bar{\omega}^{\prime}(\zeta)$ into (2.7) gives

$$
a \Phi(\zeta, \bar{\zeta})=e^{-\zeta}\left[\Omega^{\prime}(\zeta)-\Omega_{U}^{\prime}(\bar{\zeta}+2 i \alpha)\right]+\left(e^{-\bar{\zeta}}-e^{2 i \alpha-\zeta}\right) \Omega^{\prime \prime}(\bar{\zeta}) .
$$

When $\zeta=\xi+i a$, it is easily seen from (2.6) that

$$
\Omega^{\prime}(\xi+i \alpha)-\Omega_{v}^{\prime}(\xi+i \alpha)=a e^{\xi+i \alpha} f(\xi) .
$$

Similarly, let the analytic continuation of $\Omega(\zeta)$ into $S^{L}$ be given by

$$
\Omega_{L}^{\prime}(\zeta)=-e^{-2 i \alpha} \Omega^{\prime \prime}(\zeta+2 i \alpha)-\bar{\omega}^{\prime}(\zeta+2 i \alpha),
$$

for $\zeta$ in $S^{L}$. Hence, replacing $\zeta+2 i \alpha$ by $\bar{\zeta}$,

$$
\bar{\omega}^{\prime}(\bar{\zeta})=-e^{-2 i \alpha} \bar{\Omega}^{\prime \prime}(\bar{\zeta})-\Omega_{L}^{\prime}(\bar{\zeta}-2 i \alpha),
$$

for $\zeta$ in $S$. Hence, from (2.7) and the second of (2.6),

$$
\Omega^{\prime}(\xi-i \alpha)-\Omega_{L}^{\prime}(\xi-i \alpha)=a e^{\xi-i \alpha} g(\xi) .
$$

Now $\omega(\zeta)$ is a single valued function in $S$, and, therefore, the two definitions (2.9) and (2.12) are identical, giving, on replacing $\xi$ by $\zeta$, the differential-difference equation

$$
\Omega_{U}^{\prime}(\zeta+2 i \alpha)-\Omega_{L}^{\prime}(\zeta-2 i \alpha)+2 i \sin 2 \alpha{\overline{\Omega^{\prime \prime}}}^{\prime}(\zeta)=0,
$$


for $\zeta$ in $S$. The functional relations (2.10), (2.13) and (2.14) are sufficient to obtain the general solution of the boundary value problem.

\section{The Eigenfunctions of the Wedge}

The eigenfunctions are obtained by considering the complementary function of the linear equations (2.10), (2.13), (2.14). Take $f(\xi)=g(\xi)=0$, when it is easily seen that

$$
\Omega_{U}^{\prime}(\zeta)=\Omega_{L}^{\prime}(\zeta)=\Omega^{\prime}(\zeta)
$$

and the system reduces to the homogeneous equation

$$
\Omega^{\prime}(\zeta+2 i \alpha)-\Omega^{\prime}(\zeta-2 i \alpha)+2 i \sin 2 \alpha \bar{\Omega}^{\prime \prime}(\zeta)=0 .
$$

Assume that

$$
\left.\Omega^{\prime}(\zeta)=\begin{array}{ll}
0\left(e^{c_{1} \xi}\right), & \xi \rightarrow \infty, \\
0\left(e^{-c_{2} \xi}\right), & \xi \rightarrow-\infty,
\end{array}\right\}
$$

where $c_{1}, c_{2}$, are positive numbers. Then the function $\phi_{+}(u), u=t+i s$, defined by

$$
\phi_{+}(u)=\frac{1}{2 \pi} \int_{0}^{\infty} \Omega^{\prime}(\zeta) e^{i u \zeta} d \zeta,
$$

is analytic for $s>c_{1}$, and $\phi_{-}(u)$, defined by

$$
\phi_{-}(u)=\frac{1}{2 \pi} \int_{-\infty}^{0} \Omega^{\prime}(\zeta) e^{i u \zeta} d \zeta,
$$

is analytic for $s<-c_{2}$. Also,

$$
\Omega^{\prime}(\zeta)=\int_{i \gamma_{1}-\infty}^{i \gamma_{1}+\infty} \phi_{+}(u) e^{-i u \zeta} d u+\int_{-i \gamma_{2}-\infty}^{-i \gamma_{2}+\infty} \phi_{-}(u) e^{-i u \zeta} d u,
$$

where $\gamma_{1}>c_{1}$, and $\gamma_{2}>c_{2}$. Substitution of (3.6) in (3.2) gives

$$
\begin{aligned}
\int_{i \gamma_{1}-\infty}^{i \gamma_{1}+\infty}\left[\phi_{+}(u) \sinh 2 \alpha u+u \Phi_{+}(-u) \sin 2 \alpha\right] e^{-i u \xi} d u \\
\quad+\int_{-i \gamma_{2}-\infty}^{-i \gamma_{2}+\infty}\left[\phi_{-}(u) \sinh 2 \alpha u+u \Phi_{-}(-u) \sin 2 \alpha\right] e^{-i u \zeta d u}=0 .
\end{aligned}
$$

Following Titchmarsh [6], the necessary and sufficient conditions for (3.7) to hold are that

$$
\begin{aligned}
\phi_{-}(u) \sinh 2 \alpha u+u \Phi_{-}(-u) \sin 2 \alpha & =-\phi_{+}(u) \sinh 2 \alpha u-u \Phi_{+}(-u) \sin 2 \alpha, \\
& =X_{R}(u),
\end{aligned}
$$


where $X_{R}(u)$ is an arbitrary function which is analytic in the infinite rectangle $R,-c_{2}<s<c_{1}$. Taking the complex conjugate of (3.8), replacing $u$ by $-u$, and eliminating $\Phi_{-}(-u), \Phi_{+}(-u)$, we have

$$
\phi_{-}(u)=-\phi_{+}(u)=\frac{X_{R}(u) \sinh 2 \alpha u+u \bar{X}_{R}(-u) \sin 2 \alpha}{\sinh ^{2} 2 \alpha u-u^{2} \sin ^{2} 2 \alpha} .
$$

If $X_{R}(u)=Y_{R}(u)+Z_{R}(u)$, where

$$
Y_{R}(u)=-\bar{Y}_{R}(-u) \text {, and } Z_{R}(u)=Z_{R}(-u),
$$

then it can be shown from (3.6) and (3.9) that

$$
\Omega^{\prime}(\zeta)=\oint \frac{Y_{R}(u)}{\sinh 2 \alpha u+u \sin 2 \alpha} e^{-i u \zeta} d u+\oint \frac{Z_{R}(u)}{\sinh 2 \alpha u-u \sin 2 \alpha} e^{-i u \zeta} d u,
$$

where the path of integration is anti-clockwise around the boundary of the rectangle $R,-c_{2}<s<c_{1}$. It is assumed that $Y_{R}(u), Z_{R}(u)$, are such that contributions to the integral from the short sides of the rectangle are vanishingly small.

\section{(a) The symmetric eigenfunctions}

For symmetry about the $x$ axis, $D(z, \bar{z})=D(\bar{z}, z)$, and $\Phi(z, \bar{z})=\Phi(\bar{z}, z)$, whence $\Omega^{\prime}(z)=\bar{\Omega}^{\prime}(z)$, and, therefore, $\Omega^{\prime}(\zeta)=\bar{\Omega}^{\prime}(\zeta)$. It can be shown in this case that $X_{R}(u)=-\bar{X}_{R}(-u)$, so that the first integral in (3.11) gives the symmetric eigenfunctions. Taking

$$
\Omega^{\prime}(\zeta)=\oint \frac{Y_{R}(u)}{\sinh 2 \alpha u+u \sin 2 \alpha} e^{-i u \zeta} d u,
$$

the singularities of the integrand are poles at $u=0, i \beta_{n}$, where $\beta_{n}=\lambda_{n} / 2 \alpha$, and the $\lambda_{n}$ are the roots of the transcendental equation

$$
\sin \lambda+k \lambda=0,
$$

where $k=\sin 2 \alpha / 2 \alpha$. The properties of this equation and its roots have been studied in detail by Smith-White and Buchwald [7]. In general the roots are simple, and are located symmetrically about the real and imaginary axes. There may be a single double root at a discrete set of values $k^{(i)}$ of $k\left(k^{(i)}=-\cos \lambda^{(i)}\right.$, where $\lambda^{(i)}$ are the roots of $\tan \lambda=\lambda$ ). For a given value of $k \neq 0$, there will be a finite number of pairs of real roots, and an infinite sequence of quadruplets of complex roots. The latter have the asymptotic form, as $n \rightarrow \infty$,

$$
\pm l_{n} \pm i \log 2|k| l_{n}+0(\log n / n)
$$

where $l_{n}=\left(2 n-\frac{1}{2}\right) \pi$ if $k>0$, and $l_{n}=\left(2 n+\frac{1}{2}\right) \pi$ if $k<0$. 
Suppose that there are $N$ positive real roots of (3.13), denoted by $\lambda_{1} \cdots \lambda_{N}$, and let $\lambda_{n}$ be the $n$-th root if it is real, i.e. $n \leqq N$. If $n>N$, $\lambda_{n}$ will refer to the appropriate complex conjugate pair $\lambda_{n}, \bar{\lambda}_{n}$, with positive real part, whose asymptotic form is given by (3.14). Allowing $c_{1}, c_{2}$, to tend to infinity, the integral in (3.12) is replaced by the expansion

$$
\begin{aligned}
\Omega^{\prime}(\zeta)=A_{0} & +\sum_{n=1}^{N}\left(A_{n} e^{-\beta_{n} \zeta}+B_{n} e^{\beta_{n} \zeta}\right) \\
& +\sum_{N+1}^{\infty}\left(A_{n} e^{-\beta_{n} \zeta}+A_{n} e^{-\bar{\beta}_{n} \zeta}+B_{n} e^{\beta_{n} \zeta}+\bar{B}_{n} e^{\bar{\beta}_{n} \zeta}\right) .
\end{aligned}
$$

In order to obtain (3.15), we have used the fact that $\bar{Y}(-u)=-Y(u)$. Hence, for $0 \leqq n \leqq N, \bar{Y}\left(-i \beta_{n}\right)=-Y\left(i \beta_{n}\right)$, and, as the $\beta_{n}$ are real

$$
A_{n}=2 \pi i Y\left(i \beta_{n}\right) /\left(2 \alpha \cos \lambda_{n}+\sin 2 \alpha\right) \text {, }
$$

so $A_{n}$ is real. A similar argument shows that for $n>N$, if $A_{n}$ is the coefficient corresponding to $\lambda_{n}$, then $\bar{A}_{n}$ is the coefficient which corresponds to $\bar{\lambda}_{n}$. Transforming back to the $z$ plane and integrating,

$$
\begin{aligned}
\Omega(z) & =A_{0} \log (z / a)+\sum_{n=1}^{N}\left[C_{n}(z / a)^{-\beta_{n}}+D_{n}(z / a)^{\beta_{n}}\right] \\
& +\sum_{N+1}^{\infty}\left[C_{n}(z / a)^{-\beta_{n}}+\bar{C}_{n}(z / a)^{-\bar{\beta}_{n}}+D_{n}(z / a)^{\beta_{n}}+\bar{D}_{n}(z / a)^{\bar{\beta}_{n}}\right],
\end{aligned}
$$

where $A_{0}, C_{n}, D_{n}$ are arbitrary constants, which are real for $n \leqq N$, and $\mathscr{R}\left(\beta_{n}\right)>0$ for $n>N$.

If $\lambda_{N}=\lambda_{N-1}=\lambda^{(i)}$, one of the roots of $\tan \lambda=\lambda$, then the integrand in (3.13) has a double pole, and the terms in $\beta_{N}, \beta_{N-1}$, would have to be replaced by

$$
\left(C_{N-1}+C_{N} \log z\right) z^{-\beta^{(i)}}+\left(D_{N-1}+D_{N} \log z\right) z^{\beta^{(i)}} .
$$

The first term in (3.16) corresponds to the well known case of an isolated load acting at the vertex of the wedge, along the axis of symmetry, leaving the faces of a wedge of any angle free of stress. The terms with the $C_{n}$ as coefficients are regular as $z \rightarrow \infty$, and singular at the vertex, while the terms with the $D_{n}$ as coefficients are regular at $z=0$, and singular as $z \rightarrow \infty$.

The complete expansion which is regular at the origin is given by the terms in $D_{n}$. However, in order that the stresses shall be regular at $z=0$, it is necessary for $\Omega^{\prime}(z), z \Omega^{\prime \prime}(z)$, to be finite there, i.e. $\mathscr{R}\left(\beta_{n}\right) \geqq 1$. Now if $\alpha \leqq \frac{1}{2} \pi, k>0$, and then the first root $\lambda_{1}$ of (3.13) can be shown to have a real part which is not less than $\pi$, so that $\mathscr{R}\left(\beta_{1}\right) \geqq 1$. Thus for a semivertical angle $\alpha \leqq \frac{1}{2} \pi$, there is no stress singularity at the origin. However 
if $\alpha>\frac{1}{2} \pi$, then $\lambda_{1}<\pi$, and $\beta_{1}<1$. Hence, for $\alpha>\frac{1}{2} \pi$, there is a stress singularity of $0\left(\gamma^{\beta_{1}-1}\right)$ at the origin. For convenience, the graph of $\beta_{1}$ is given for the range $0 \leqq \alpha \leqq \pi$, in Fig. 2 .

The Airy stress function $\chi$ is defined in terms of the complex potentials by

$$
\chi=\mathscr{R}\left[\tilde{z} \Omega(z)+\int \omega(z) d z\right] .
$$

After some computation, it can be shown that the complete symmetric expansion (3.16), together with (2.12), (3.13), and (3.17), gives the expansion in polar coordinates

$$
\chi=A_{0}^{\prime} r \theta \sin \theta+\sum_{\beta_{n}} A_{n}^{\prime} r^{\beta_{n}+1} H_{n}(\theta),
$$

where

$$
H_{n}(\theta)=\cos \left(\beta_{n}+1\right) \theta \cos \left(\beta_{n}-1\right) \alpha-\cos \left(\beta_{n}-1\right) \theta \cos \left(\beta_{n}+1\right) \alpha,
$$

the summation is over all the roots of (3.13), and the $A_{n}^{\prime}$ are arbitrary constants derived from the $C_{n}$ and $D_{n}$.

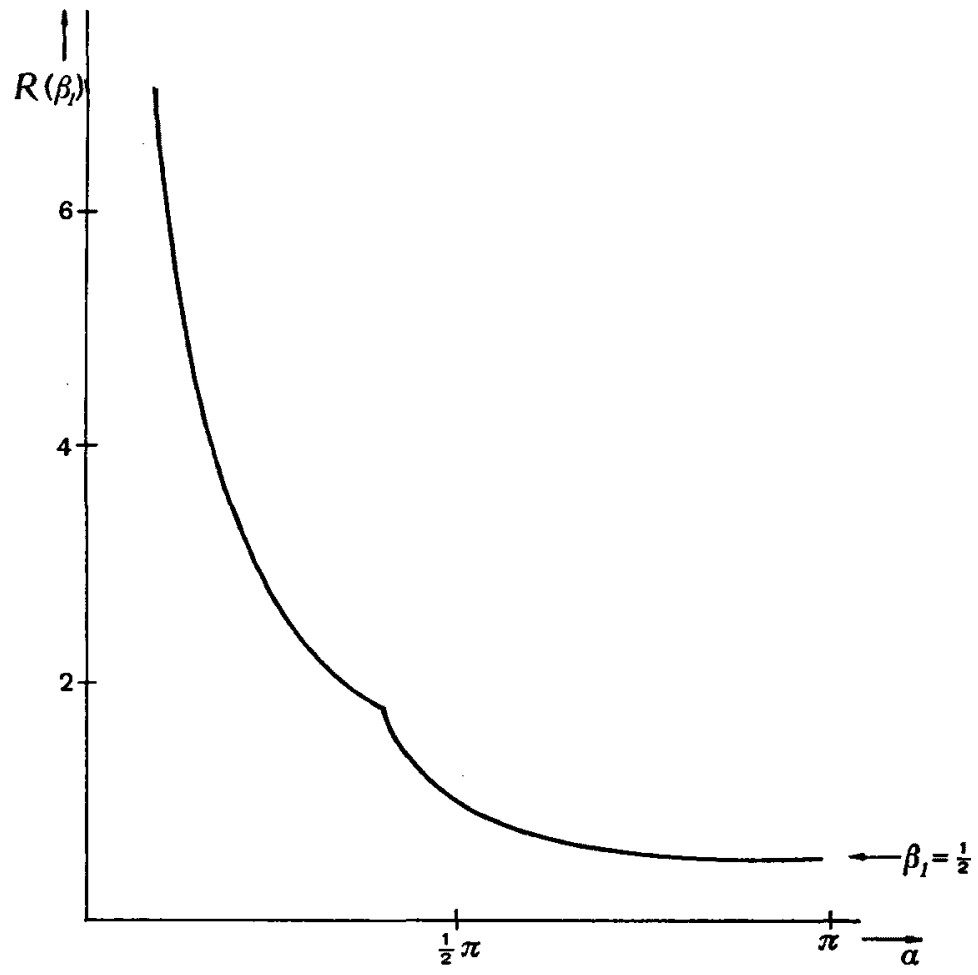

Fig. 2. Graph of the real part of the first root $\beta_{1}$ of the equation $\sin 2 \alpha \beta+\beta \sin 2 \alpha=0$, for values of $\alpha$ in the range $0<\alpha \leqq \pi$. 


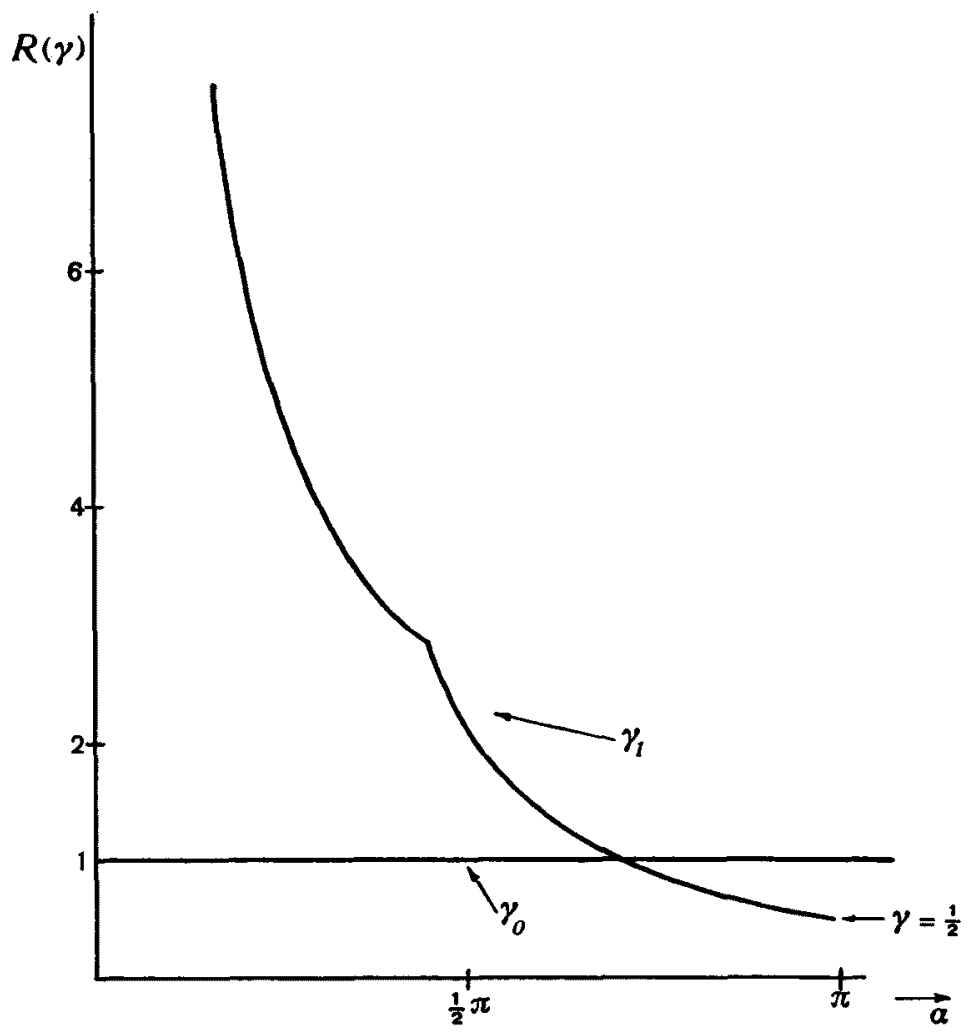

Fig. 3. Graph of the real parts of the first two roots $\gamma_{0}, \gamma_{1}$, of the equation $\sin 2 \alpha \gamma-\gamma \sin 2 \alpha=0$, for values of $\alpha$ in the range $0<\alpha \leqq \pi$.

(b) The anti-symmetric case.

In this case $X_{R}(u)=\boldsymbol{X}_{R}(-u)$, and, therefore,

$$
\Omega^{\prime}(\zeta)=\oint \frac{Z_{R}(u)}{\sinh 2 \alpha u-u \sin 2 \alpha} e^{-i u \zeta d u}
$$

and the expansion is in terms of the roots of the equation

$$
\sin \mu-k \mu=0 \text {. }
$$

This equation has real roots at $\mu=0, \pm 2 \alpha$, and at $\mu=\mu_{1}, \cdots, \mu_{N}$, and complex roots at $\pm \mu_{n}, \pm \tilde{\mu}_{n}$ for $n>N$. The asymptotic form for the complex $\mu_{n}$ for $k \neq 0$ is

$$
\mu_{n}=l_{n}+i \log 2|k| l_{n}+0(\log n / n),
$$

where now $l_{n}=\left(2 n+\frac{1}{2}\right) \pi$ if $k>0$, and $l_{n}=\left(2 n-\frac{1}{2}\right) \pi$ if $k<0$. 
If $\gamma_{n}=\mu_{n} / 2 \alpha$, the poles of the integrand in (3.19) are at $u=0, \pm i$, $\pm i \gamma_{n}$ for $n \leqq N$; $\pm i \bar{\gamma}_{n}, \pm i \gamma_{n}$ for $n>N$. The contributions from the three poles at $u=0, \pm i$, are of the form

$$
\Omega_{0}^{\prime}(\zeta)=i A_{0}-i B_{0} e^{-\zeta}+i C_{0} e^{\zeta},
$$

where $A_{0}, B_{0}, C_{0}$, are real constants. Changing the variable and integrating,

$$
\Omega_{0}(z)=i A_{0} \log (z / a)+i B_{0} a / z+i C_{0} z / a
$$

whence

$$
\omega_{0}(z)=i A_{0} \log (z / a)+2 i B_{0} a \cos 2 \alpha / z .
$$

The term in $C_{0}$ represents a rigid body rotation, and, without loss of generality, we take $C_{0} \equiv 0$. The term in $A_{0}$ represents an isolated load in the $y$ direction at the origin, and the term in $B_{0}$ corresponds to an isolated couple, also at $z=0$.

Thus the complete anti-symmetric eigenfunction expansion is

$$
\begin{aligned}
\Omega(z) & =\Omega_{0}(z)+i \sum_{n=1}^{N}\left[E_{n}(z / a)^{-\gamma_{n}}+F_{n}(z / a)^{\gamma_{n}}\right] \\
& +\sum_{N+1}^{\infty}\left[E_{n}(z / a)^{-\gamma_{n}}-E_{n}(z / a)^{-\bar{\gamma}_{n}}+F_{n}(z / a)^{\gamma_{n}}-\bar{F}_{n}(z / a)^{\bar{\gamma}_{n}}\right],
\end{aligned}
$$

where the $E_{n}, F_{n}$ are arbitrary constants, real for $n \leqq N$, and $\mathscr{R}\left(\gamma_{n}\right)>0$ for $n>N$. The equivalent expansion of $\chi$ in polar coordinates is

$$
\chi=A_{0}^{\prime} r \theta \cos \theta+B_{0}^{\prime}(\sin 2 \theta-2 \theta \cos 2 \alpha)+\sum_{\gamma_{n}} A_{n}^{\prime} r_{n+1} H_{n}(\theta),
$$

where now

$$
H_{n}(\theta)=\sin \left(\gamma_{n}+1\right) \theta \sin \left(\gamma_{n}-1\right) \alpha-\sin \left(\gamma_{n}-1\right) \theta \sin \left(\gamma_{n}+1\right) \alpha .
$$

The exceptional case is when $\mu_{N}, \mu_{N-1}$, are roots of $\tan \lambda=\lambda$, and there is a pole of order two. The appropriate terms in the expansion (3.23) are of the form

$$
i\left(E_{N-1}+E_{N} \log z\right) z^{-\gamma_{N}}+i\left(F_{N-1}+F_{N} \log z\right) z^{\gamma_{N}} .
$$

Even more exceptional is the case where $\alpha$ is the first non-trivial root $\alpha_{0}$ of $\tan 2 \alpha=2 \alpha$, where $0<\alpha_{0}<\pi$. In this case it is easily seen that the double poles are at $u= \pm i$, and the first terms in the expansion of $\Omega(z)$ in (3.23) are

$$
\Omega_{0}(z)+i \log z\left[E_{1}(a / z)+F_{1}(z / a)\right]+\cdots .
$$

The stress singularity at the origin has somewhat different properties than in the symmetric case. In (3.23), the expansion for which $\Omega(z)$ is 
finite at the origin is the one associated with the $F_{n}$, while the $E_{n}$ give an expansion which is regular as $z \rightarrow \infty$. Thus, in the case of the expansion which gives finite $\Omega(z)$ at $z=0$, the stresses are finite there only if $\mathscr{R}\left(\gamma_{1}\right)>1$. It is easily seen that this inequality holds for $\alpha \leqq \frac{1}{2} \pi$. If $\alpha>\frac{1}{2} \pi$, however, the equation

$$
\sin 2 \alpha \gamma-\gamma \sin 2 \alpha=0
$$

has two real zeros in the range $\pi \leqq 2 \alpha \gamma \leqq 2 \pi$. One of these is $\gamma=1$, and we denote the other by $\gamma_{1}$. Now if $\alpha_{0}$ is the first root of $\tan 2 \alpha=2 \alpha$, $\left[\alpha_{0} \approx 3 \pi / 4\right]$, and if $\alpha<\alpha_{0}, \gamma_{1}>1$, and there is no stress singularity. However, if $\alpha>\alpha_{0}$, the two roots change places, and $\gamma_{1}<1$, so that there is a stress singularity of $0\left(r^{\gamma_{1}-1}\right)$ at the vertex. If $\alpha=\alpha_{0}$, then $\gamma_{1}=1$, the two roots are equal, and $F_{1}$ is the coefficient of a term in $z \log z$, so that $\Omega^{\prime}(z) \sim \log z$ at the origin. We conclude that in the antisymmetric case there is a singularity at the origin only if $\alpha \geqq \alpha_{0}$.

The behaviour of the first two roots of (3.26) is illustrated in Fig. 3. Noting that $\gamma_{0}=1$ is always a root of (3.26), we see from the graphs that $\gamma_{0}$ is the smallest root if $\alpha<\alpha_{0}$, while if $\alpha>\alpha_{0}, \gamma_{0}$ becomes the second root, and $\gamma_{1}$ is the smallest root.

The question of the concentrated couple corresponding to the coefficient $B_{0}$ has caused some discussion in previous works. For example, Sternberg and Koiter [8] have reported on the existence of a 'paradox'. It would appear from the above analysis that there is no paradox at all, but that the existence of the term in $B_{0}$ is part of the eigenfunction structure of the differential operator. At $\alpha=\alpha_{0}$ two eigenvalues coincide, and there is a confluence of eigenfunctions, illustrated by the double pole in the integrand in (3.19). Note also that at $\alpha=\frac{1}{2} \pi$, the poles at $t=i$ in the symmetric and antisymmetric cases coincide. However, a glance at (3.9) shows that this is a highly degenerate case, and, since the wedge here is simply a half-plane, it does not need any further discussion.

The 'paradox' reported by Sternberg and Koiter was obtained by taking the limit of certain loads on the faces of the wedge. The question as to whether this limit is strictly applicable will be discussed at the end of the paper.

\section{The Orthogonality Property}

In polar coordinates, $\chi$ satisfies the equation

$$
\left(\frac{\partial^{2}}{\partial r^{2}}+\frac{1}{r} \frac{\partial}{\partial r}+\frac{1}{r^{2}} \frac{\partial^{2}}{\partial \theta^{2}}\right)^{2} \chi=0 .
$$

Assuming that 


$$
\chi=r^{1+\beta} H(\theta),
$$

it is easily seen that $H(\theta)$ satisfies

$$
\frac{d^{4} H}{d \theta^{4}}+2\left(1+\beta^{2}\right) \frac{d^{2} H}{d \theta^{2}}+\left(\beta^{2}-1\right)^{2} H=0,
$$

subject to the boundary conditions

$$
H=\frac{d H}{d \theta}=0
$$

at $\theta= \pm \alpha$, for all values of $\beta \neq 0, \pm 1$. The symmetric and antisymmetric solutions of (4.2) and (4.3) are given after equations (3.18) and (3.24), respectively.

Let $\beta_{m}, \beta_{n}$, be two distinct, eigenvalues, and let $H_{m}, H_{n}$, be the corresponding functions of $\theta$, with $\chi_{n}=r^{1+\beta_{n}} H_{n}$. It can easily be shown (e.g. [5]) that if neither $\beta_{m}, \beta_{n}$, take the values $0, \pm 1$, and $\beta_{m} \neq \beta_{n}$, then

$$
\int_{-\alpha}^{\alpha}\left[H_{m} \frac{d^{2} H_{n}}{d \theta^{2}}+H_{n} \frac{d^{2} H_{m}}{d \theta^{2}}+\left(\beta_{m}^{2}+\beta_{n}^{2}-2\right) H_{m} H_{n}\right] d \theta=0 .
$$

This integral can be rewritten in the form

$$
\left[\chi_{n}, \chi_{m}\right]=\int_{-\alpha}^{\alpha}\left[\chi_{n} \Delta \chi_{m}+\chi_{m} \Delta \chi_{n}\right] d \theta=0,
$$

where $\Delta$ is the operator

$$
\Delta \equiv r \frac{\partial}{\partial r}\left(\frac{1}{r} \frac{\partial}{\partial r}\right)+\frac{1}{r^{2}} \frac{\partial^{2}}{\partial \theta^{2}}
$$

Unifortunately, although it is interesting, the property (4.5) is not generally suitable for the solution of physical boundary value problems. For instance, consider a sectorial region bounded by the straight lines $\theta= \pm \alpha$, and the arc $r=a$. If $\tau_{\theta \theta}=\tau_{r \theta}=0$ on $|\theta|=\alpha$, the suitable eigenfunction expansions are those in (3.18) and (3.24) with $\mathscr{R}\left(\beta_{n}\right)$ and $\mathscr{R}\left(\gamma_{n}\right)$ positive, so that displacements are finite at $r=0$. The boundary conditions at $r=a$ are

$$
\tau_{r r}=\frac{1}{r^{2}} \frac{\partial^{2} \chi}{\partial \theta^{2}}+\frac{1}{r} \frac{\partial \chi}{\partial r} ; \quad \tau_{r \theta}=-\frac{\partial^{2}}{\partial r \partial \theta}\left(\frac{\chi}{r}\right) ;
$$

are given functions of $\theta$ for $|\theta|<\alpha$. By suitable combinations of these conditions, it is possible to obtain $\chi$ as a function of $\theta$ on $r=a$. The same is not true for $\Delta \chi$, and, as in [5], indirect means have to be used to evaluate the coefficients of the expansions. 
Note, however, that the constants $A_{0}^{\prime}$ in (3.18), and $A_{0}^{\prime}, B_{0}^{\prime}$, in (3.24) can be specifically determined by evaluating the resultant force and moment on the circular boundary, as, for static equilibrium, these must be balanced by an equal and opposite isolated load and couple at the vertex.

\section{Deformation of a Wedge, Loaded on its Faces}

This case has been dealt with before, both by Tranter [1], and Green and Zerna [2], but there are a number of features which are of further interest. We, therefore, find a particular integral of the system of equations (2.10), (2.13), and (2.14). As the stresses on the faces of the wedge are not necessarily in static equilibrium, they must be balanced by forces and moments, either at the vertex, or in the wedge interior at infinity, or both. Assume for the time being that there is no isolated load or couple at the vertex, but that at infinity, $\Omega(z)$ is such that the resultant force and moment there balance the loads on the faces. Thus, assume that

$$
\Omega(z)=0\left(z^{\sigma}\right), \text { as } z \rightarrow 0 ; \Omega(z)=0(\log z), \text { as } z \rightarrow \infty ;
$$

where $\sigma\left(\geqq \frac{1}{2}\right)$ is the positive real part of the first nontrivial root of

$$
\sin 2 \alpha \lambda \pm \lambda \sin 2 \alpha=0,
$$

and $\sigma=\frac{1}{2}$ when $\alpha=\pi$. (See Figs. 2 and 3.) Hence

$$
\left.\Omega(\zeta)=\begin{array}{ll}
0\left(e^{\sigma \xi}\right), & \xi \rightarrow-\infty, \\
0(\xi), & \xi \rightarrow \infty .
\end{array}\right\}
$$

In making these assumptions, it is understood that in (2.4), $f_{0}(r)$, $g_{0}(r)$, are $o\left(r^{-1}\right)$, as $r \rightarrow \infty$, otherwise it would not be possible to balance the loads by a finite resultant at infinity. Thus, in $(2.6), f(\xi), g(\xi)$, are $0\left(e^{-\xi}\right)$, as $\xi \rightarrow \infty$, and, from $(2.10)$ and (2.13), this is consistent with the assumption that $\Omega(\zeta)=0(\xi)$ as $\xi \rightarrow \infty$.

On account of (5.2), the Fourier integral

$$
\phi(u)=\frac{1}{2 \pi} \int_{-\infty}^{\infty} \Omega^{\prime}(\zeta) e^{i u \zeta} d \zeta, \quad u=t+i s,
$$

is an analytic function of $u$ in the strip $0<s<\sigma$, and, therefore,

$$
\Omega^{\prime}(\zeta)=\int_{i c-\infty}^{i c+\infty} \phi(u) e^{-i u \zeta} d u,
$$

where $0<c<\sigma$. Note that if we had assumed that the loads were balanced by forces and moments at the vertex, we would need to assume that $\Omega(z)=o\left(z^{-1}\right)$ as $z \rightarrow \infty$, when the path of integration in (5.4) would be taken below the singularity at $u=-i$. It is clear that the choice of taking 
the path of integration above or below the singularities at $u=0,-i$, does not affect the conditions on the faces of the wedge, but determines just where the equilibrating forces are acting, at the vertex or at infinity. If the loads on the faces are in equilibrium, the singularities at $u=0,-i$, will be removable, and there will be no resultants at the vertex, or infinity.

It will be assumed for the remainder of the paper that there are no isolated forces or moments at the vertex. It will also be assumed that $\alpha$ is such that the equations (3.13) and (3.20) do not have a double root. In so far as the double roots are concerned, those only occur for transcendental values of $\alpha$, and are of no concern from the physical point of view. If desired, the results which will be obtained can easily be amended to take into account any double roots which might occur.

The Fourier Transforms $\phi_{U}(u), \phi_{L}(u)$ of the functions $\Omega_{U}^{\prime}(\zeta), \Omega_{L}^{\prime}(\zeta)$, are defined as in (5.3), and let

$$
\begin{aligned}
& 2 \pi F(u)=\int_{-\infty}^{\infty} e^{\xi(1+i u)} f(\xi) d \xi, \\
& 2 \pi G(u)=\int_{-\infty}^{\infty} e^{\xi(1+i u)} g(\xi) d \xi .
\end{aligned}
$$

The transforms of equation (2.10) and (2.13) are

$$
\begin{aligned}
& \phi(u)-\phi_{U}(u)=a e^{-\alpha(u-i)} F(u), \\
& \phi(u)-\phi_{L}(u)=a e^{\alpha(u-i)} G(u) .
\end{aligned}
$$

The transformation of equation (2.14) gives

$$
e^{2 \alpha u} \phi_{U}(u)-e^{-2 \alpha u} \phi_{L}(u)+2 u \bar{\phi}(-u) \sin 2 \alpha=0 .
$$

On eliminating $\phi_{U}(u), \phi_{L}(u)$, from these equations, we have

$$
\phi(u) \sinh 2 \alpha u+u \bar{\phi}(-u) \sin 2 \alpha=H(u),
$$

where

$$
2 H(u)=a e^{a(u+i)} F(u)-a e^{-\alpha(u+i)} G(u) .
$$

Taking the conjugate of (5.9), replacing $u$ by $-u$, and eliminating $\bar{\phi}(-u)$, we have

$$
\left(\sinh ^{2} 2 \alpha u-u^{2} \sin ^{2} \alpha\right) \phi(u)=H(u) \sinh 2 \alpha u+u \bar{H}(-u) \sin 2 \alpha .
$$

If the applied loads are symmetric about the axis, then $g_{0}(r)=f_{0}(r)$ in $(2.4)$, and $g(\xi)=\bar{f}(\xi)$. Hence, from (5.5), $G(u)=\vec{F}(-u)$, so that in (5.9), $H(u)=-H(-u)$. Thus, for symmetric loading,

$$
(\sinh 2 \alpha u+u \sin 2 \alpha) \phi(u)=H(u) \text {. }
$$


Similarly, for anti-symmetric loads, $g(\xi)=-\bar{f}(\xi)$, and then $\bar{H}(-u)=H(u)$, and

$$
(\sinh 2 \alpha u-u \sin 2 \alpha) \phi(u)=H(u) .
$$

In the general case in (5.10), the integrand $\phi(u)$ in (5.4) has singularities at all the zeros of

$$
\sinh ^{2} 2 \alpha u-u^{2} \sin ^{2} 2 \alpha=0,
$$

as well as at possible singularities of $H(u)$, depending on the nature of the loads. In general, it will be possible to expand the integral in terms of the singularities of the integrand, to give eigenfunction expansions in appropriate sections of the wedge.

As an example, we obtain a useful Green's function by considering the case, as in Fig. 4, of a normal isolated load $P$ at the point $z=a e^{i \alpha \text {, }}$ on the upper face. This can be represented by taking

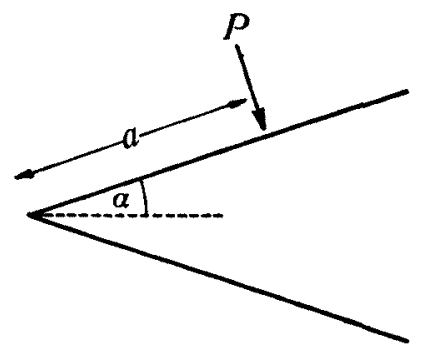

Fig. 4. An isolated normal load $P$ at $z=a e^{\imath a}$.

$$
f_{0}(r)=-P \delta(r-a) ; \quad g_{0}(r)=0 ;
$$

where $\delta(r-a)$ is the Dirac delta function. If $r=a e^{5}$, this gives

$$
a f(\xi)=-P e^{-\xi} \delta(\xi) ; \quad g(\xi)=0 ;
$$

whence, from (5.5),

$$
2 \pi a F(u)=-P ; \quad G(u)=0 ;
$$

and, therefore,

$$
4 \pi H(u)=-P \exp \alpha(u+i)
$$

Hence

$$
4 \pi \Omega^{\prime}(\zeta)=-P \int_{i \sigma-\infty}^{i \sigma+\infty} \frac{e^{\alpha(u+i)} \sinh 2 \alpha u+u e^{-\alpha(u+i)} \sin 2 \alpha}{\sinh ^{2} 2 \alpha u-u^{2} \sin ^{2} 2 \alpha} e^{-i u \zeta} d u .
$$

When $\xi>0$ (i.e. $r>a$ ), the integral can be replaced by the negative sum 
of the residues at the poles in the half plane $s<c$, and when $\xi<0$, (i.e. $r<a$ ), the integral can be replaced by the sum of the residues at the poles in the half plane $s>c$.

Considering first of all the residues at $u=0,-i$, these give, on transformation to the $z$ plane,

$\Omega_{0}(z)=-\frac{1}{2} P\left[\frac{\sin \alpha}{\sin 2 \alpha+2 \alpha}+\frac{i \cos \alpha}{\sin 2 \alpha-2 \alpha}\right] \log z+\frac{1}{2} i P(2 \alpha \cos 2 \alpha-\sin 2 \alpha)^{-1} \frac{a}{z}$.

This expression corresponds to a resultant force $-i P e^{i \alpha}$ and moment $a P$ being carried along the wedge from $r=a$ to infinity.

Evaluating the residues at the other poles in $s<\sigma$, it can be shown that for $r \geqq a$

$$
\Omega(z)=\Omega_{0}(z)-\frac{1}{4} i P \sum_{R\left(\lambda_{n}\right)>0} \frac{e^{i \alpha\left(1-\lambda_{n}\right)} \sin 2 \alpha \lambda_{n}+\lambda_{n} e^{-i \alpha\left(1-\lambda_{n}\right)} \sin 2 \alpha}{\left(\alpha \sin 4 \alpha \lambda_{n}-\lambda_{n} \sin ^{2} 2 \alpha\right) \lambda_{n}}\left(\frac{a}{z}\right)^{\lambda_{n}},
$$

where the $\lambda_{n}$ are the roots of the equation

$$
\sin ^{2} 2 \alpha \lambda-\lambda^{2} \sin ^{2} 2 \alpha=0,
$$

and the summation is over all the roots to the right of the imaginary axis, except $\lambda=1$.

Similarly, for $r<a$, the pole at $u=i$ gives $\Omega_{1}(z)=i P(\tan 2 \alpha-2 \alpha)^{-1} z$, and, summing over the remaining poles,

$$
\Omega(z)=\Omega_{1}(z)+\frac{1}{4} i P \sum_{\mathscr{R}\left(\lambda_{n}\right)>0} \frac{e^{i \alpha\left(1+\lambda_{n}\right)} \sin 2 \alpha \lambda_{n}+\lambda_{n} e^{-i \alpha\left(1+\lambda_{n}\right)} \sin 2 \alpha}{\left(\alpha \sin 4 \alpha \lambda_{n}-\lambda_{n} \sin ^{2} 2 \alpha\right) \lambda_{n}}\left(\frac{z}{a}\right)^{\lambda_{n}} .
$$

The expressions (5.19) and (5.21) are suitable for rapid calculations by digital computer, since, for a given value of $\alpha$, the solutions of $(5.20)$ can be found by the complex form of Newton's method. If the expressions (3.14) and (3.21) are used to obtain a first estimate, only a few iterations are necessary to obtain the roots of $(5.20)$ to a high degree of accuracy.

However, the radius of convergence of the series in (5.19) and (5.21) is $a$, and the series are divergent at $r=a e^{i \alpha}$, and only slowly convergent at other points on the circle $r=a$. In general, in order to evaluate quantities at $r=a$, the best policy appears to be interpolation between values found for $r>a$ and $r<a$. Near the singularity at $r=a e^{i \alpha}$, however, even this method fails unless the singular part is first removed. It is easy to show that the singular part of $\Omega(z)$ near $z=z_{0}=a e^{i \alpha}$ is

$$
2 \pi \Omega_{s}(z)=i P e^{i \alpha} \log \left(z-z_{0}\right), \quad|\arg z|<\alpha .
$$

The 'divergent' part of the expansion (5.21) can be shown to be 


$$
-\frac{1}{4} i P \sum \frac{e^{i \alpha}}{\alpha \lambda_{n}}\left(\frac{z}{z_{0}}\right)^{\lambda_{n}},
$$

where the summation is over all the roots in the quadrant

$$
0>\arg \lambda>-\frac{1}{2} \pi \text {. }
$$

Enumerating the roots in this quadrant, as $n \rightarrow \infty$, we see that the general term in this expansion

$$
\sim-\frac{i P e^{i \alpha}}{2 n \pi}\left(\frac{z}{z_{0}}\right)^{n \pi / \alpha},
$$

which is equivalent to the expansion of the singular part of $\Omega_{8}(z)$ in the form

$$
\Omega_{s}(z)=-\frac{i P e^{i \alpha}}{2 \pi} \sum_{n=1}^{\infty} n^{-1}\left(\frac{z}{z_{0}}\right)^{n \pi / \alpha} .
$$

Thus by writing $\Omega^{*}(z)=\Omega(z)-\Omega_{s}(z)$, and subtracting (5.23) term by term from (5.21), better convergence is obtained. A similar method may be used to improve the convergence of the series in (5.19) near the singularity.

It is quite clear from the above analysis that any loads on the faces of the wedge give rise to appropriate eigenfunction expansions, and there is no evidence that a particular loading configuration can pick out a single eigenvalue. The loading taken by Sternberg and Koiter [8] is statically equivalent to a couple, and the eigenfunction expansion will contain a term corresponding to a couple at infinity, as well as the other terms ir: the expansion. There is no reason to believe that, in the limit, the loading taken by them must reduce to an isolated couple, which is a single term in the eigenfunction expansion. It appears, in fact, that there is no 'paradox', other than the slow decay of the stresses as $r \rightarrow \infty$, when $\alpha>\frac{1}{2} \pi$. If $\alpha>\frac{1}{2} \pi$ in the symmetric case, and $\alpha>\alpha_{0}$ in the antisymmetric case, the decay of the stresses is less than $0\left(r^{-1}\right)$, as $r \rightarrow \infty$. However, insofar as the stresses due to the tip of a semi-infinite crack decay as $0\left(r^{-\frac{1}{2}}\right)$, this behaviour is not really surprising.

Department of Applied Mathematics, University of Sydney.

\section{References}

[1] C. J. Tranter, Q.J.M.A.M. 1 (1948), 125.

[2] A. E. Green and W. Zerna, Theoretical Elasticity, 1954, Oxford University Press.

[3] G. F. Carrier and F. S. Shaw, Proc. Symp. App. Math. 3 (1950), 125. 
[4] L. S. D. Morley, Q.J.M.A.M. 15 (1962), 413.

[5] V. T. Buchwald, Proc. Roy. Soc. A, 277 (1964), 385.

[6] E. C. Titchmarsh, Fourier Transforms, Oxford University Press, 1948.

[7] W. B. Smith-White and V. T. Buchwald, this Journal 4 (1964), 327.

[8] E. Sternberg and W. T. Koiter, J. Appl. Mech. 4 (1958), 575. 